\title{
Temporary transvenous cardiac pacing: 6 years experience in one coronary care unit
}

\author{
N.I. Jowett, D.R. Thompson and J.E.F. Pohl \\ Coronary Care Unit, Leicester General Hospital, Leicester LE5 4PW, UK.
}

\begin{abstract}
Summary: The role of temporary percutaneous endocardial pacing has been examined in a retrospective analysis of all paced patients admitted to one coronary care unit over a 6 year period. The majority of 162 cases (84.6\%) were paced for complete heart block complicating acute myocardial infarction. These patients had a higher incidence of previous hypertension, myocardial infarction and diabetes, compared to matched controls $(P<0.05,<0.02$ and $<0.001$, respectively). Admission blood glucose levels were also higher $(P<0.05)$. The in-hospital mortality was high $(46.7 \%)$, especially for those with anterior myocardial infarction $(\mathbf{7 4 . 5 \% )}$.

Twenty-five (15.4\%) patients without recent myocardial infarction were paced for symptomatic brady-dysrhythmias, usually due to chronic complete heart block (Lenegre's disease) or sick sinus syndrome. Most later required permanent pacing. Complications of temporary pacing were more frequent in those who died, the most common being dysrhythmias during pacemaker insertion.

Review of our cases suggests that whilst facilities for temporary pacing were extremely valuable, many cases treated were not haemodynamically compromised and probably did not require pacing. Guidelines should be established on coronary care units to prevent the unnecessary morbidity, mortality and expense of the procedure.
\end{abstract}

\section{Introduction}

Temporary endocardial pacing has been used in acute myocardial infarction since the early 1960 s to maintain cardiac output during episodes of extreme bradycardia, heart block and asystole. Before the advent of cardiac pacemakers, the combination of acute myocardial infarction and complete heart block was usually fatal, ${ }^{1}$ but now pacing electrodes can be safely passed percutaneously into the right ventricle under local anaesthesia. There is little doubt that pacing may be life saving in the short term, but long term prognosis is a little more difficult to assess. Prognosis is influenced not only by complications of the procedure (e.g. dysrhythmias, cardiac perforation, septicaemia), but also by the underlying myocardial damage which originally lead to the conduction defect. Many patients effectively treated by acute temporary pacing die from heart failure whilst still in hospital. ${ }^{2}$

In patients who have not suffered an acute myocardial infarction, temporary pacing may be required for treatment of chronic atrio-ventricular block, sicksinus syndrome and other special categories. For

Correspondence: N.I. Jowett, M.D., M.R.C.P.

Accepted: 28 November 1988 example, patients with sino-atrial disease or incomplete heart block may be at risk of developing complete heart block (CHB) during drug therapy or surgery; general anaesthesia with fluorinated hydrocarbons may adversely affect atrio-ventricular conduction. ${ }^{3}$

Previous studies have concentrated on the evolution and prognosis of heart block following acute myocardial infarction ${ }^{4,5}$ and the role of temporary pacing has not been considered since the early small series. ${ }^{2,6,7} \mathrm{We}$ have therefore reviewed our practice to ascertain the role which temporary cardiac pacing plays in a typical coronary care unit by studying 4,791 serial admissions to our unit, including 2,065 patients with confirmed acute myocardial infarction, and report our experience.

\section{Materials and methods}

The coronary care unit at Leicester General Hospital is an 8 bedded purpose built unit which accepts 999 calls and direct admissions from general practititioners on a 24 hour basis. Patients are rapidly assessed on admission with attention focused on relief of pain

(C) The Fellowship of Postgraduate Medicine, 1989 
and haemodynamic stabilization. The need for artificial pacing is assessed by the duty coronary care house officer, and is accomplished via the sub-clavian vein using a bipolar electrode catheter fixed to a demand pacemaker pack. A side room adjacent to the coronary care unit is fully equipped for insertion of temporary pacing wires, with resuscitation facilities and an image intensifier to aid quick and stable positioning of the electrodes. A log-book is kept for all pacings containing clinical details of the patients. The notes of all patients who had temporary pacing wires inserted over a 6-year period were examined retrospectively. Details regarding personal data, previous medical history, diagnosis and clinical course were noted.

Patients who had sustained acute myocardial infarctions were classed as primarily anterior or inferior infarctions, depending upon electrocardiographic evidence. An age-sex matched control group of 137 patients with acute myocardial infarction at similar sites, but who had no conduction problems was also studied.

\section{Results}

During the study period there were 4,791 admissions to the coronary care unit, and 162 patients had temporary pacing wires inserted.

\section{Patients with myocardial infarction}

Two thousand and sixty-five patients had confirmed myocardial infarction by standard criteria of clinical history with serial electrocardiographic and serum enzymes changes; ${ }^{8} 137(6.6 \%)$ of these had temporary pacing wires inserted; 90 patients with inferior infarction $(66 \%)$ and 47 with anterior infarction $(34 \%)$.

There was a higher incidence of previous diabetes mellitus, myocardial infarction and hypertension in patients who were paced than in our control group (Table I). Admission blood glucose levels were higher in patients who were paced than in those who were not, particularly in those who later died.

Forty-nine per cent of these 137 patients were admitted in CHB, and within 24 hours of admission $61 \%$ were affected. The remainder developed CHB over the next 5 days, and most developed disturbances of conduction before its onset. In those with inferior infarction, 1st degree and Mobitz type 1 heart block were common, whereas in anterior infarction, heart block was usually sudden and preceded by Mobitz type 2 or bifascicular block (right bundle branch block with left anterior hemi-block in 3 cases and with left posterior hemi-block in one case). There was no difference in the time of onset of heart block and the location of the infarct.

The time which the wires remained in situ varied from 1 hour to 11 days (mean 2.6 days), although pacing wires were not taken out immediately after the return of sinus rhythm; a safety period of up to 24 hours was usually allowed before removal. Pacing requirements were longer in patients with anterior infarction (mean 4.1 days), and in those patients who died (mean 3.6 days).

Two thirds of the pacing deaths occurred within 24 hours of admission, and most $(89 \%)$ within 72 hours. The unit mortality for those patients with inferior myocardial infarction was $32 \%$ and those with anterior infarction was $74 \%$.

Table I Clinical details of paced patients with acute myocardial infarction

\begin{tabular}{lcccc}
\hline & Deaths & Survivors & Totals & Controls \\
\hline No: & 64 & 73 & 137 & 137 \\
M/F: & $50 / 14$ & $57 / 16$ & $107 / 30$ & $107 / 30$ \\
Mean years age & & & & \\
$\quad$ (range): & $63.6(47-84)$ & $57.3(42-71)$ & $60.5(42-84)$ & $60.5(42-84)$ \\
Previous & & & & $27(20 \%)$ \\
$\quad$ Hypertension: & 18 & 20 & $38(28 \%)$ & $30(22 \%)$ \\
$\quad$ Infarction: & 29 & 15 & $44(32 \%)$ & $12(9 \%)$ \\
$\quad$ Diabetes: & 14 & 20 & $26(19 \%)$ & 47 \\
Ant. infarct: & 35 & 12 & 47 & 90 \\
Inf. infarct: & 29 & 61 & $10.5 \pm 4.9$ & $6.1 \pm 2.4$ \\
BGL*: & $12.3 \pm 6.1$ & $8.3 \pm 4.7$ & & \\
\hline
\end{tabular}

\footnotetext{
* Mean blood glucose level mmol/l ( \pm s.d.), excluding known diabetics. Comparison with matched controls by $t$-test: $P<0.05$. Comparison by chi-square analysis of paced patients versus controls for previous hypertension, myocardial infarction and diabetes: $P<0.05,<0.02,<0.001$ respectively.
} 


\section{Non-infarction cases}

Table II shows details of the remaining 25 patients who were paced because of symptomatic bradyarrhythmias; $18(72 \%)$ of these were later transferred to another hospital for electro-physiological assessment and subsequent implantation of permanent pacemakers.

Table II Non-infarction patients who were paced

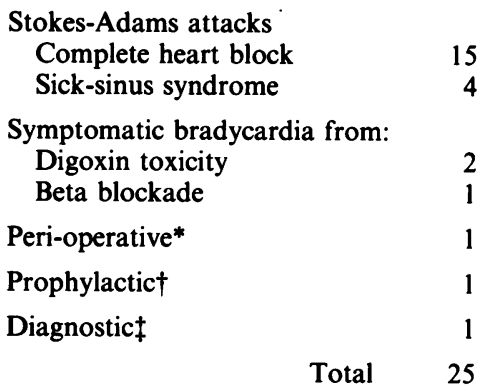

* Patient with intermittent Mobitz type 1 heart block.

$\dagger$ Patient with recurrent ventricular tachycardia, right bundle branch block and $1^{\circ}$ heart block: inserted during antidysrhythmic drug therapy.

$\ddagger$ Differentiation between recurrent supra-ventricular and ventricular tachycardia.

\section{Complications}

Complications associated with pacemaker insertion occurred in 32 cases (19.8\%), and were more common in those who died (Table III). Insertion dysrhythmias included supraventricular tachycardia, ventricular tachycardia and ventricular fibrillation. Most responded to withdrawal of the catheter tip, but defibrillation was required in 5 cases and was successful in all but one case.

Repositioning of the pacing wire was needed in 12 cases because of spontaneously displaced electrodes with pacing failure.

Only two small pneumothoraces were noted related to subclavian catheterization. Neither needed treatment.

Possible perforation of the heart was seen at postmortem in three cases, but not suspected at the time of death. Two of these had extensive myocardial infarction and rupture of the heart. In the remaining one patient there was a $200 \mathrm{ml}$ bloody pericardial effusion. It was not possible to determine whether catheter perforation was responsible for death in any of these cases. No clinical evidence of perforation was noted in any other case.
Table III Complications of temporary pacings in myocardial infarction

\begin{tabular}{lccc}
\hline Complication & Deaths & Survivors & $\begin{array}{c}\text { \% of all } \\
\text { cases paced }\end{array}$ \\
\hline Insertion dysrhythmias & 13 & 2 & 9.2 \\
Repositioning & 5 & 7 & 7.4 \\
Pneumothorax & 1 & 1 & 1.2 \\
Perforated heart & 3 & $?$ & 1.8 \\
\hline
\end{tabular}

\section{Discussion}

CHB complicates $9-33 \%$ of acute myocardial infarctions. ${ }^{4-6}$ Only $6.3 \%$ of our patients were paced following acute myocardial infarction which may imply that some cases of $\mathrm{CHB}$ were managed without temporary pacing.

Transient conduction disturbances commonly follow acute inferior myocardial infarction, and the benefits of routine pacing for inferior myocardial infarction are doubtful. Mortality was $32.3 \%$ in our paced patients, and has been reported as $37 \%$ without pacing. ' $\mathrm{CHB}$ developed slowly and the escape rhythm was usually high junctional at $40-60$ beats/minute, and generally haemodynamically stable. Pacing is unnecessary, provided blood pressure and renal perfusion are maintained. ${ }^{6}$ The decision by the duty senior house officer to insert a pacing wire often appeared to be routine, although previous studies would suggest that the majority of cases do not deteriorate, and pacing is not necessary. ${ }^{6}$

CHB in patients with anterior myocardial infarction was associated with extensive myocardial damage, heart failure and cardiogenic shock. Onset was sudden, with slow and irregular escape ventricular pacemakers. Whilst insertion of a temporary pacing wire under these circumstances probably had little influence on the outcome, it is our usual practice. Mortality was high in this group (74.5\%), and late deaths might be expected in those who survive. ${ }^{9}$ Prophylactic wires have been advocated in those who develop atrioventricular or interventricular block in the presence of anterior myocardial infarction, ${ }^{10.11}$ Of the 12 patients with myocardial infarction in whom prophylactic wires were inserted, half developed complete heart block (Table IV). Antero-septal myocardial infarction with right bundle branch block (RBBB) had a poor prognosis $(67 \%)$, regardless of whether $\mathrm{CHB}$ developed and was associated with cardiogenic shock and refractory heart failure. This supports the findings of previous studies. ${ }^{2}$

Hypertension, pre-existing diabetes mellitus and high admission blood glucose concentrations were common predisposing factors for those developing 
Table IV Insertion of prophylactic wires; $8.7 \%$ of wires were inserted prophylactically for conduction defects complicating myocardial infarction

\begin{tabular}{lccc}
\hline Problem & $N$ & $C H B$ & Died \\
\hline RBBB $/ 1^{\circ}$ HB & 5 & $2^{*}$ & $3(60 \%)$ \\
RBBB/LAHB & 6 & 3 & $4(67 \%)$ \\
RBBB/LPHB & 1 & 1 & $1(100 \%)$ \\
Totals & 12 & 6 & $8(67 \%)$ \\
\hline
\end{tabular}

All infarctions were anterior apart from *2 inferior infarctions.

RBBB = right bundle branch block; LAHB = left anterior hemi-block; LPHB = left posterior hemi-block; $1^{\circ} \mathrm{HB}=$ first degree AV block; $\mathrm{CHB}=$ complete heart block.

CHB as a complication of myocardial infarction. Czyzyk $^{12}$ found that disorders of atrio-ventricular and intra-ventricular conduction were significantly more common in diabetic patients with acute myocardial infarction than those who were not diabetic, and this may be due to pre-existing micro-angiopathic damage of the conducting system. ${ }^{13}$ So called 'stress' hyperglycaemia of greater than $10 \mathrm{mmol} / \mathrm{l}$ on admission to coronary care may represent pre-existing diabetes, ${ }^{14}$ and the contribution which undiagnosed diabetes makes to the mortality of acute myocardial infarction is therefore probably underestimated.$^{15}$ There was also a higher incidence of previous myocardial infarctions and hypertension in those patients requiring pacing, probably indicating a greater degree of underlying myocardial damage.

Most non-infarction cases who were paced had Stokes-Adams attacks due to chronic CHB. The underlying disease is usually Lenegre's disease, and since the minority are due to coronary heart disease, the prognosis is much better. ${ }^{16}$ Four patients were found to have the sick sinus syndrome, and, although many of such cases have a co-existing cardiomyopathy, there were no deaths. Temporary pacing was

\section{References}

1. Cohen, D.B., Doctor, L. \& Pick, A. The significance of atrioventricular block complicating myocardial infarction. Am Heart J 1958, 55: 215-219.

2. Norris, R.M., Mercer, C.J.\& Croxson, M.S. Conduction disturbances due to anteroseptal myocardial infarction and their treatment by endocardial pacing. Am Heart $J$ 1972, 84: 560-566.

3. Atlee, J.L. \& Alexander, S.C. Halothane effects on conductivity of the atrioventricular node and HisPurkinje system in the dog. Anesth Analg 1977, 56: 378-386. also of value in the acute management of poisoning by digoxin and beta-adrenergic blocking agents.

Complications of pacing were more common in patients who subsequently died, and the most frequent was ventricular fibrillation which occurred in $15.6 \%$ of cases who were paced. Most dysrhythmias terminated on withdrawal of the tip of the wire, and were probably produced by mechanical irritation of the myocardium. Provided immediate defibrillation and resuscitation facilities are available, immediate recovery is good.

This study confirms the need for the availability of pacing facilities, but argues a more convincing case for an effective medical staff rota for emergency pacemaker insertion than the widespread use of prophylactic pacing. The marginal reduction of mortality in a high risk sub-group of patients with myocardial infarction must be related to the $19.8 \%$ incidence of pacing complications and the relative unpleasantness of the procedure. It is likely that at least some of the complications are due to inexperience of the medical staff and are thus preventable. The more benign nature of CHB complicating inferior myocardial infarction would imply that such cases should be paced only in response to haemodynamic necessity and that prophylactic pacing should not be routinely used. The very high mortality of anterior myocardial infarction complicated by CHB would make pacing mandatory since most patients are haemodynamically disturbed, and it is not possible to ascertain what contribution a low heart rate makes to the clinical picture withouto effectively removing it. ${ }^{11,17}$ Further arguments in? favour of such a policy are the small numbers involved and the desirability of determining the optimum heart rate in seriously haemodynamically compromised patients.

Since it is usually junior doctors who make the immediate decision to pace or not, firm guidelines should be established on units to prevent the unnecessary morbidity, mortality and expense associated with the procedure.

4. Norris, R.M. Heart block in posterior and anterior myocardial infarction. Br Heart $J$ 1969, 31: 352-356.

5. Kostuk, W.J. \& Beanlands, D.S. Complete heart block associated with myocardial infarction. Am J Cardiol 1970, 26: 380-384.

6. Gupta, P.K., Lichstein, E. \& Chadda, K.D. Heart block complicating acute inferior wall myocardial infarction. Chest 1976, 69: 599-604.

7. Lassers, B.W. \& Julian, D.G. Artificial pacing in the management of complete heart block complicating acute myocardial infarction. $\mathrm{Br} \mathrm{Med} J$ 1968, 2: 142-146. 
8. Rowley, J.M. \& Hampton, J.R. Diagnostic criteria for myocardial infarction. $\mathrm{Br} J$ Hosp Med 1981, 18: 253-258.

9. Mullins, C.B. \& Atkins, J.M. Prognoses and management of ventricular conduction blocks in acute myocardial infarction. Mod Concepts Cardiovasc Dis 1976, 45: 129-133.

10. Hindman, M.C., Wagner, G.S., Jaro, M. et al. The clinical significance of bundle branch block complicating acute myocardial infarction. Circulation 1978, 58: 679-699.

11. DeGuzman, M. \& Rahimtoola, S.H. What is the role of pacemakers in patients with coronary heart disease and conduction abnormalities. In: Rahimtoola, S.H. (ed.) Controversies in Coronary Artery Disease. F.A. Davis Co., Philadelphia, 1983, pp. 191-207.

12. Czyzyk, A., Krolewski, A.S., Szablowska, S., Alot, A. \& Kopczynski, J. Clinical course of myocardial infarction amongst diabetic patients. Diabetes Care 1980, 4: $526-529$.
13. Blandford, R.L. \& Burden, A.C. Abnormalities of cardiac conduction in diabetics. $\mathrm{Br} \mathrm{Med} J$ 1984, 289: 1659.

14. Husband, D.J., Alberti, K.G. \& Julian, D.G. 'Stress' hyperglycaemia during acute myocardial infarction: an indicator of pre-existing diabetes? Lancet 1983, ii: 179-181.

15. Oswald, G.A., Corcoran, S. \& Yudkin, J.S. Prevalence and risks of hyperglycaemia and undiagnosed diabetes in patients with acute myocardial infarction. Lancet 1984, i: $1265-1267$.

16. Hayward, R. Who do we pace? Br J Hosp Med 1981, 25: $466-474$.

17. Klein, R.C., Vera, Z. \& Mason, D.T. Intra-ventricular conduction defects in acute myocardial infarction: Incidence, prognosis and therapy. Am Heart $J$ 1984, 4: $1007-1013$. 